\section{\$28. Three-Dimensional Simulation Study of Flux Rope Dynamics in the Solar Corona}

Kusano, K. (The Earth Simulator Center, JAMSTEC), Inoue, S. (Solar Terrestrial Environment Laboratory, Nagoya University)

Solar flares and coronal mass ejections (CMEs) are the biggest explosion in our solar system, and greatly influence the electromagnetic condition around the earth, resulting magnetospheric storms, sub-storms, and the onset of aurora burst. Even though both flares and CMEs are widely believed to be related to magnetic energy liberation by magnetic reconnection, the physical relationship between flare and CMEs is not yet well understood.

On the other hand, it is often observed that many flares were associated with the eruption or the disappearance of dark filaments, which existed above magnetic neutral line until the onset of events. Therefore, it is also an important issue to understand whether is there some causality between the filament eruption and the onset of flares and CMEs. Various models have been proposed to explain the filament eruption mechanism.

However, the several important questions are not yet solved. Which in the loss-of-equilibrium or the loss-of-stability is more important for the launching of CME? What is the condition for filament eruption to develop to CME, and to be failed as a so-called confined eruption. In order to give the answers to them, the nonlinear 3D numerical simulation has been performed.1)

The simulation domain is a rectangle box, $(0,0,0)$ $<(\mathrm{x}, \mathrm{y}, \mathrm{z})<(\mathrm{Lx}, \mathrm{Ly}, \mathrm{Lz})$, in which the magnetic neutral line is parallel to the $\mathrm{x}$ axis, and $\mathrm{z}$ denotes the altitude from the photospheric surface. The initial state consists of a magnetic arcade equilibrium, in which a force-free flux rope is embedded at $\mathrm{z}=\mathrm{h}$, plus a small perturbation that is given by the eigenfield of kink mode instability for the equilibrium. The equilibrium arcade is given by a dipole field for the magnetic dipole located at $\mathrm{z}=-\mathrm{d}$ as same as the model proposed by Forbes and Priest.2) The horizontal boundaries for the $\mathrm{x}$ and $\mathrm{y}$ coordinate satisfy the periodic condition, and the top boundary $(\mathrm{z}=\mathrm{Lz})$ is located far enough from the filament height $(\mathrm{h}<<\mathrm{Lz})$.

Several calculations have been carried out for various value of $\mathrm{Lx}$ that corresponds to the length of flux rope. As a result of them, it was found that there is a threshold of Lx for the flux rope to be able to escape out of the arcade. For instance, when $L x=7.5 \mathrm{~d}$, the flux rope can ascend continuously even after the growth phase of kink instability, as shown in Fig.1, whereas, for $\mathrm{Lx}=3 \mathrm{~d}$, the upward motion of the flux rope stops at a certain height.

Even for small Lx, however, we found that, if some external force is artificially applied onto the flux rope to help the upward motion only for a limited period before the ascending is saturated, the ascending of the flux rope can be continued. The result implies that there is some critical height, which has to be exceeded for the flux rope to escape from the confinement by magnetic arcade.
Also the variation of plasma density structure is investigated, and it was found that plasma density is depressed at the top of the magnetic rope during the ascending phase due to the cross-field expansion, as shown in Fig.2. The structure of the density depression is consistent with the observation of dimming in CMEs. The all results suggest the possibility that the kink instability is involved in the launching of CMEs.

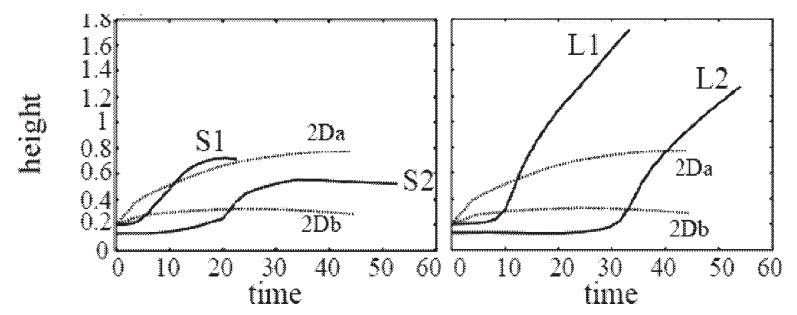

Fig. 1. The time history of the flux rope height for the various runs. Left and right panels show the result for $\mathrm{Lx}=3 \mathrm{~d}$ and $7.5 \mathrm{~d}$, respectively. For $\mathrm{S} 1$ and $\mathrm{L} 1$, the loss-of-equilibrium state is used as the initial state, and for S2 and L2 the dipole magnetic field is stronger than them. $2 \mathrm{Da}$ and $2 \mathrm{Db}$ are the results of the two-dimensional simulations, in which the translational symmetry for the $\mathrm{x}$ coordinate is applied.

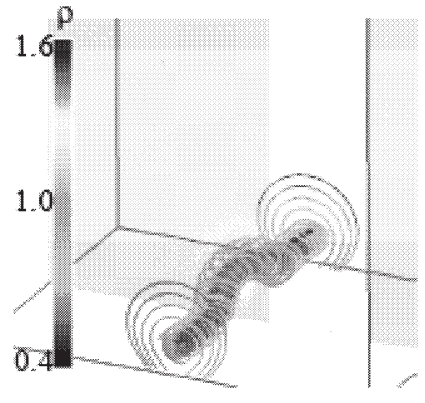

TIME $=9.42$

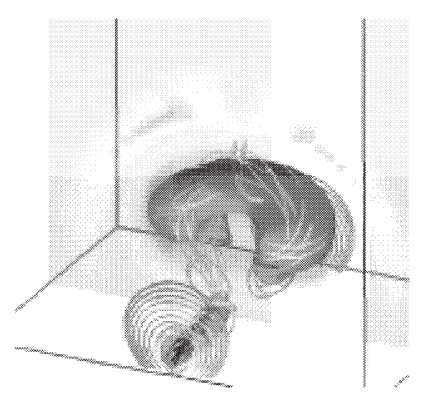

TMME $=14.80$

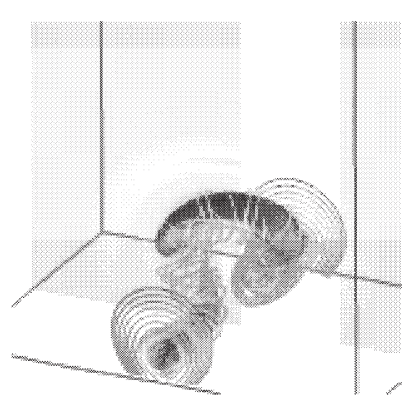

TIME $=12.11$

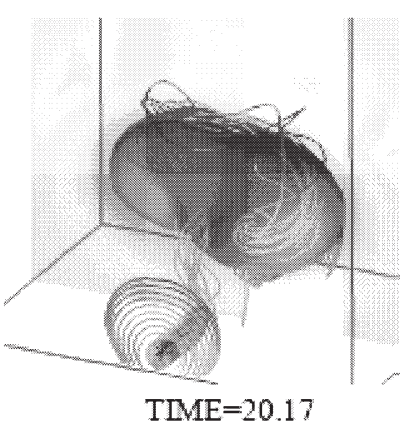

Fig. 2. The evolution of magnetic flux rope in case of $\mathrm{Lx}=7.5 \mathrm{~d}$. The gray surface is an iso-surface of density representing the density depression region.

References

1) Inoue, S., Kusano, K., The Astrophys. J. 645, (2006) 742

2) Forbes, T., Priest, E., The Astrophys. J. 446, (1995) 377 